\title{
Hyperbaric oxygen therapy for the treatment of anastomotic complications after tracheal resection and reconstruction
}

\author{
Cameron Stock, MD, Natalie Gukasyan, BS, Ashok Muniappan, MD, Cameron Wright, MD, and \\ Douglas Mathisen, MD
}

\begin{abstract}
Objective: Failure of anastomotic healing is a rare but serious complication of laryngotracheal resection. Treatment options include reoperation, tracheostomy, or T-tube placement. Hyperbaric oxygen therapy (HBOT) is the delivery of $100 \% \mathrm{O}_{2}$ at pressures greater than $1 \mathrm{~atm}$, and has been shown to enhance wound healing after tracheal resection in animal models. To date, there have been no reports describing its usefulness in humans after tracheal resection.
\end{abstract}

\begin{abstract}
Methods: Five consecutive patients with varying degrees of failed anastomotic healing, from necrotic cartilage to partial separation identified by bronchoscopy were treated with HBOT. HBOT was administered for $90 \mathrm{mi}-$ nutes via a hyperbaric chamber pressurized to 2 atm with $100 \%$ oxygen. Patients were treated with daily or twice daily HBOT. Four of 5 patients had buttressing of the anastomosis by strap muscle at the initial surgery.
\end{abstract}

Results: All patients had evidence of anastomotic healing on bronchoscopy. None of the patients in this series required tracheostomy, T-tube, or reoperation after initiation of HBOT. On average it took 9.6 days for healing to occur (5-14 days). The size of the anastomotic defect ranged between 3 and $13 \mathrm{~mm}$. One patient required bilateral tympanostomy tubes for inner ear discomfort and experienced blurry vision as complications of HBOT. One patient developed tracheal stenosis from granulation tissue that required bronchoscopic debridement.

Conclusions: In select patients with anastomotic complications after tracheal resection, HBOT may aid in healing and avoid tracheostomy. Future investigations are necessary to further define the benefits of HBOT in the management of airway anastomotic complications. (J Thorac Cardiovasc Surg 2014;147:1030-5)

Anastomotic separation after tracheal resection and reconstruction (TRR) is rare, occurring in only $4 \%$ of procedures. ${ }^{1}$ However, anastomotic separation carries significant morbidity and a $0.6 \%$ chance of mortality. ${ }^{1}$ Risk factors for anastomotic complications include diabetes, resection length greater than $4 \mathrm{~cm}$, reoperation, laryngotracheal resections, age less than 17 years, and preoperative tracheostomy. ${ }^{1} \mathrm{We}$ have previously described our experience with anastomotic complications after TRR. Treatment of anastomotic complications included reoperation, revision of the anastomosis, and T-tube or tracheostomy placement. ${ }^{1}$

Hyperbaric oxygen therapy (HBOT) is the administration of $100 \%$ oxygen at pressures greater than $1 \mathrm{~atm}$. It is currently used to increase healing of diabetic lower extremity wounds and radiation injuries. HBOT is believed to promote wound healing by increasing angiogenesis and collagen

From the General Thoracic Surgical Unit, Department of Surgery, Massachusetts General Hospital, Harvard Medical School, Boston, Mass.

Disclosures: Authors have nothing to disclose with regard to commercial support.

Read at the 39th Annual Meeting of The Western Thoracic Surgical Association, Coeur d'Alene, Idaho, June 26-29, 2013.

Received for publication June 25, 2013; revisions received Oct 9, 2013; accepted for publication Nov 8, 2013; available ahead of print Dec 16, 2013.

Address for reprints: Douglas Mathisen, MD, Division of Thoracic Surgery, Massachusetts General Hospital, Harvard Medical School, Boston, MA (E-mail: dmathisen@partners.org).

$0022-5223 / \$ 36.00$

Copyright (c) 2014 by The American Association for Thoracic Surgery

http://dx.doi.org/10.1016/j.jtcvs.2013.11.014 synthesis. ${ }^{2}$ Recent experimental evidence in animals has shown that it may stimulate increased healing of the trachea after resection and reconstruction. ${ }^{3,4}$ We used HBOT in 5 consecutive patients with anastomotic complications after tracheal resection and followed their clinical outcomes.

\section{METHODS}

This is a retrospective review of 5 consecutive patients treated at a single institution from 2009 to 2012. Approval for this study was obtained from the Institutional Review Board. Each patient previously underwent a tracheal resection and anastomosis and was found to have evidence of failed anastomotic healing on bronchoscopic examination between postoperative days 5 and 7 . Our technique for tracheal and laryngotracheal resection has been described in previous publications.${ }^{5-8}$ Bronchoscopic findings ranged from necrotic cartilage to partial separation of the tracheal anastomosis. All patients were treated as inpatients and HBOT was administered for 90 minutes via a hyperbaric chamber pressurized to 2 atm with $100 \%$ oxygen. The goal number of HBOT treatments was 20 . Initially in our series, patients were treated with daily HBOT, however later patients received twice daily HBOT. Four of 5 patients in this series had careful buttressing of the primary anastomosis with sternohyoid and sternothyroid strap muscles. One patient with anastomotic separation did not undergo buttressing of the anastomosis with muscle and underwent reexploration, revision of the anastomosis, and tracheostomy placement. In this patient, HBOT was started after the reoperation procedure. All patients received intravenous broad-spectrum antibiotics and 3 of 5 patients also received treatment with tobramycin nebulizers administered twice daily. Heliox was used in patients as clinically indicated.

\section{RESULTS}

The characteristics of the patients in this series were as follows: the mean age was 46 years (range, 20-63 years), 

Abbreviations and Acronyms
$\mathrm{CT}=$ computed tomography
HBOT $=$ hyperbaric oxygen therapy
$\mathrm{POD}=$ postoperative day
$\mathrm{TRR}=$ tracheal resection and reconstruction

3 patients had idiopathic tracheal stenosis, 2 patients had postintubation tracheal stenosis, 3 patients were female, and 1 patient was a reoperation (Table 1). Two patients with anastomotic complications in this series had diabetes and 1 had a preoperative tracheostomy (Table 1). The clinical presentation of anastomotic complications varied (Table 2). Two patients had cervical erythema, 3 patients presented with subcutaneous air, 1 had fever, 2 had leukocytosis, 1 had stridor, and 1 had hemoptysis. Overall, 4 patients had 2 or more clinical symptoms at the time the anastomotic complication was recognized.

On bronchoscopic examination, all the patients had evidence of necrosis at the anastomotic site. Three patients had defects of the anterior cartilage; 2 patients had necrosis at the lateral cartilaginous aspect of the anastomosis. The range in size of the anastomotic defect was estimated to be between 3 and $13 \mathrm{~mm}$ (Table 2). Two patients had a significant separation of the anastomosis visible on bronchoscopy (Table 2). Two patients had positive bacterial cultures, the other 3 patients either did not have cultures or their tracheal cultures demonstrated normal respiratory flora (Table 2).

At the time of discharge, all patients underwent bronchoscopy and had evidence of anastomotic healing. None of the patients in this series required reoperation after initiation of HBOT. On average it took 9.6 days for healing to occur (range, 5-14 days) (Table 3). The mean number of HBOT sessions was 13.2 (range, 5-21) administered either daily or twice daily (Table 3). Patients in this series had on average 2.6 bronchoscopies (range, 1-4) performed after the initiation of HBOT and before discharge (Table 3). The mean interval between bronchoscopies was 5 days (range, 3-8 days) (Table 3). The average length of stay was 22 days (range, 15-31 days) (Table 4).

Outcomes are listed in Table 4. There were no postoperative deaths. Complications were minor and included 1 patient who required bilateral tympanostomy tubes for inner ear discomfort. The same patient also experienced temporary blurry vision after receiving HBOT. Both of these complications resolved. Three patients developed granulation tissue at the anastomosis with 1 patient requiring 2 bronchoscopic debridements. At follow-up, ranging from 1 to 3.5 years, all patients remain asymptomatic and none have required subsequent dilation or persistent need to treat granulation tissue.

\section{Case 1}

The patient was a 52-year-old man with obesity, diabetes, coronary artery disease, gastroesophageal reflux disease, and fibromyalgia who required a tracheostomy in February 2009 after undergoing multiple nasal and pharyngeal procedures for obstructive sleep apnea. The tracheostomy site was complicated by recurrent infections. He was decannulated in May 2009. He subsequently developed stenosis at the site of his tracheostomy stoma and was referred for definitive resection and reconstruction after undergoing 2 failed dilation procedures. He had a $3-\mathrm{cm}$ long stenosis 3 $\mathrm{cm}$ below the vocal cords.

TRR was performed through a collar incision with the addition of a partial sternotomy through the manubrium to aid in exposure. The anastomosis was to the cricoid cartilage and a sternohyoid muscle flap was used to buttress the anastomosis. Postoperatively, the patient did well until postoperative day (POD) 7 when he developed erythema of the neck in addition to leukocytosis. He was placed on broad-spectrum antibiotics. Bronchoscopy was performed and persistent necrosis of the anterior cartilage at the anastomosis was observed. A repeat bronchoscopy on POD 14 revealed progression of the necrosis and visualization of the strap muscle buttress. Frank dehiscence of the anastomosis did not occur. HBOT was started that day after a computed tomography (CT) scan of the neck did not reveal subcutaneous air, abscess, or fluid collection. He completed 6 sessions of HBOT between POD 14 and 20. On POD 21, bronchoscopic examination demonstrated granulation tissue and a gathering of sutures at the anastomosis. The airway was intact, and the patient was otherwise doing well. Bronchoscopic debridement of the sutures and granulation tissue was undertaken on POD 27 and POD 31. He was discharged on POD 32. The patient remains asymptomatic 3.5 years later with mild stenosis $(20 \%)$ on bronchoscopic examination.

\section{Case 2}

The patient was a 20-year-old man who developed tracheal stenosis after tracheostomy after a motor vehicle accident. In March 2010, he underwent a tracheal reconstruction with an auricular cartilage graft and T-tube placement. The T-tube was removed but after 5 weeks, a large amount of scar and granulation tissue was noted on bronchoscopy. A tracheostomy was placed and he was referred to our institution 8 months later when it was evident that the graft had failed. Radiologic and bronchoscopic findings revealed a $3.5-\mathrm{cm}$ long narrowing that started $1.5 \mathrm{~cm}$ below the vocal cords. Tracheal resection was performed through a midline neck incision with cricotracheal anastomosis and sternohyoid muscle flap coverage.

On POD 7, bronchoscopy revealed a 3-mm region of separation and necrotic cartilage at the anterior anastomosis. A 
TABLE 1. Patient characteristics

\begin{tabular}{|c|c|c|c|c|c|}
\hline Characteristic & Patient 1 & Patient 2 & Patient 3 & Patient 4 & Patient 5 \\
\hline Diagnosis & PITS & PITS & Idiopathic & Idiopathic & Idiopathic \\
\hline Age (y) & 52 & 20 & 63 & 45 & 52 \\
\hline Sex & M & M & $\mathrm{F}$ & $\mathrm{F}$ & $\mathrm{F}$ \\
\hline Reoperation & No & Yes & No & No & No \\
\hline Diabetes & Yes & No & Yes & No & No \\
\hline $\begin{array}{l}\text { Preoperative } \\
\text { tracheostomy }\end{array}$ & No & Yes & No & No & No \\
\hline Reoperation & No & Yes & No & No & No \\
\hline $\begin{array}{l}\text { Postoperative } \\
\text { tracheostomy }\end{array}$ & No & No & No & No & No \\
\hline $\begin{array}{l}\text { Laryngotracheal } \\
\text { resection }\end{array}$ & No & No & Yes & Yes & Yes \\
\hline
\end{tabular}

PITS, Postintubation tracheal stenosis.

Gram stain taken from the anastomosis demonstrated gramnegative and gram-positive organisms, however the culture ultimately revealed normal respiratory flora. The patient was placed on inhaled tobramycin and HBOT was initiated. He completed 20 sessions between POD 8 and 21. Three subsequent bronchoscopies on POD 10, 14, and 20 showed gradual anastomotic healing. There was no purulent material present at the anastomosis after starting HBOT, and sutures that were visible on previous bronchoscopies became covered with healthy granulation tissue. He was discharged on POD 23. At 1-month follow-up, the anastomosis was well healed with only a $10 \%$ narrowing as a result of scar tissue. The patient remains asymptomatic in follow-up.

\section{Case 3}

The patient was a 63-year-old woman with diabetes, hypertension, hyperlipidemia, and depression who developed idiopathic subglottic stenosis. She had undergone multiple tracheal dilation procedures with little relief from her symptoms. A laryngotracheal resection was performed through a transverse collar incision below the cricoid and cricotracheal anastomosis was performed. Bronchoscopy performed on POD 6 revealed a widely patent, intact anastomosis with minimal vocal cord edema and minimal subglottic swelling. She was discharged home that day but returned on POD 7 with swelling and palpable subcutaneous emphysema in her neck. A CT scan demonstrated significant subcutaneous air tracking from the anastomosis to the lateral neck. A bronchoscopy performed the following day revealed a larger than expected area of anterior necrosis with anastomotic separation and visualization of the surrounding soft tissue. Based on these findings, she underwent neck reexploration whereby necrosis of the anterior portions of the first 2 rings was found. These were debrided leaving the lateral and posterior aspects of the anastomosis intact. The distal trachea was mobilized and the anterior portion was sutured to the thyroid cartilage. Strap muscles were used to cover the anastomosis and a tracheostomy was placed for airway protection. Cultures of the necrotic airway were positive for Eikenella corrodens. She subsequently completed 5 treatments of HBOT between POD 12 and 16.

Bronchoscopy was performed on POD 16 and showed moderate subglottic swelling but otherwise no necrosis or separation. The patient was discharged on POD 20. Her tracheostomy was successfully removed 1 month later and the patient has been free of complications since.

\section{Case 4}

The patient was a 45-year-old woman who was diagnosed with idiopathic subglottic stenosis after an 8-year history of shortness of breath on exertion. Her medical history also included hypertension and anxiety. She underwent 2 rigid bronchoscopies with tracheal dilation before resection and reconstruction. When her symptoms failed to improve, she underwent a TRR with cricotracheal anastomosis in May 2012. A sternohyoid strap muscle was used to buttress the anastomosis. On POD 2, the patient developed erythema

TABLE 2. Presenting symptoms and bronchoscopic findings

\begin{tabular}{|c|c|c|c|c|c|}
\hline Finding & Patient 1 & Patient 2 & Patient 3 & Patient 4 & Patient 5 \\
\hline Cervical erythema & Yes & Yes & No & Yes & No \\
\hline Subcutaneous emphysema & No & No & Yes & Yes & Yes \\
\hline Fever & No & No & No & No & Yes \\
\hline Leukocytosis & Yes & No & No & No & Yes \\
\hline Stridor & No & No & No & No & Yes \\
\hline Postoperative hemoptysis & No & Yes & No & No & No \\
\hline Location of defect(s) & Anterior & Anterior & Anterior & Lateral & Lateral \\
\hline Size of defect(s) (mm) & 10 & 3 & 10 & $\sim 8$ & $\sim 13$ \\
\hline Exudate & Yes & No & No & Yes & Yes \\
\hline Culture & Enterococcus faecalis & Normal flora & Eikenella corrodens & - & Normal flora \\
\hline Edema & No & No & Vocal cords & Anastomotic & Anastomotic \\
\hline Erythema & Yes & Yes & Yes & No & No \\
\hline Necrosis or ischemia & Yes & Yes & Yes & Yes & Yes \\
\hline Separation & No & Yes & Yes & No & No \\
\hline
\end{tabular}


TABLE 3. Treatment with HBOT

\begin{tabular}{lccccc}
\hline Treatment details & Patient 1 & Patient 2 & Patient 3 & Patient 4 & Patient 5 \\
\hline Total sessions & 6 & 20 & 5 & 14 & 21 \\
$\begin{array}{l}\text { Number of days } \\
\begin{array}{l}\text { Days until defect } \\
\text { healed }\end{array}\end{array}$ & 6 & 14 & 5 & 10 & 14 \\
$\begin{array}{c}\text { Subsequent number } \\
\text { of bronchoscopies }\end{array}$ & 3 & 12 & 5 & 9 & 14 \\
$\begin{array}{c}\text { Mean bronchoscopy } \\
\text { interval (d) }\end{array}$ & 5.7 & 4.3 & 8 & 4 & 3.2 \\
\hline
\end{tabular}

of the neck and was started on broad-spectrum antibiotics. She remained afebrile, with a normal white blood cell count, however the erythema progressed. A CT performed on POD 5 showed subcutaneous emphysema in the tissues of the neck and mediastinum (Figure 1). On the following day, bronchoscopy was performed and she was noted to have a 5-mm region of exposed cartilage covered with exudative tissue at the anastomotic site. She was started on HBOT that day, along with nebulized tobramycin and intravenous broad-spectrum antibiotics.

The patient completed 14 HBOT sessions between POD 6 and POD 15. Two days after starting HBOT, repeat bronchoscopy showed no exudate, reduced swelling, and evidence of healthy granulation starting to form at the anastomosis. By POD 9, both the erythema and subcutaneous emphysema had resolved. A bronchoscopy on POD 14 showed a well-healed, completely granulated anastomosis with minimal swelling. She was discharged home on POD 15. She remains asymptomatic 1 year later.

\section{Case 5}

The patient was a 62-year-old woman who presented with a 4-year history of shortness of breath caused by idiopathic subglottic stenosis. Multiple laser dilation procedures failed. She underwent laryngotracheal resection and reconstruction with posterior membranous tracheal wall flap and a sternohyoid muscle flap.

On POD 4, the patient developed a change in her breathing believed to be a result of laryngeal edema. She responded to a 24-hour course of steroids, but her breathing did not return to baseline. Bronchoscopy on POD 7 showed necrotic cartilage with a white exudate, 2 visible sutures, and a small 2- to 3-mm extraluminal cavity (Figure 1). She was immediately started on twice daily HBOT and nebulized tobramycin. Sputum cultures grew normal respiratory flora.

Bronchoscopy performed on POD 9 showed slight improvement in the appearance of the anastomosis, however exposed cartilage on the right side and 2 traction sutures were still visible. The rest of the airway appeared intact. On POD 14, the previously identified cavity at the anastomosis had been filled with healthy granulation tissue. There was also a significant decrease in the amount of exudate and there was new vascularized granulation tissue present. The patient developed inner ear discomfort during HBOT for which she had tympanostomy tubes placed on POD 12 and her HBOT regimen was changed to once daily. In total, she had 21 sessions between POD4 and POD 21.

A final bronchoscopy was done on POD 21 and showed that the area of necrosis had completely filled in with healing tissue. She was discharged on POD 21 and her tympanostomy tubes were discontinued. A bronchoscopy 28 days after her discharge on POD 48 demonstrated that the area of necrosis was completely healed. A small nodule of granulation tissue with mucosa covering it was noted, but there was no significant airway narrowing (Figure 1). She has remained asymptomatic at follow-up 1 year postoperatively. She previously had complained of blurry vision, which resolved 3 weeks after stopping HBOT.

\section{DISCUSSION}

We have previously reported our experience with anastomotic complications after tracheal resection. ${ }^{1}$ Our usual practice for patients with necrosis or separation of the anastomosis was to place a tracheostomy or T-tube or, in select cases, perform a revision of the anastomosis. In this study, we describe our initial experience using HBOT for the treatment of anastomotic complications after tracheal resection. To our knowledge, this is the first study to use HBOT in humans to promote tracheal healing.

Failure of anastomotic healing after TRR can result in catastrophic outcomes. ${ }^{1,5}$ When confronted with such a complication, a secure airway must be established.

TABLE 4. Outcomes

\begin{tabular}{|c|c|c|c|c|c|}
\hline Outcome & Patient 1 & Patient 2 & Patient 3 & Patient 4 & Patient 5 \\
\hline Follow-up (y) & 3.5 & 2 & 1.5 & 1 & 1 \\
\hline Granulation at the anastomosis & Yes & No & Yes & No & Yes \\
\hline Anastomotic stenosis requiring reintervention & Yes & No & No & No & No \\
\hline Separation of anastomosis that required either airway appliance or reoperation & No & No & Yes & No & No \\
\hline Length of stay (d) & 31 & 23 & 20 & 15 & 21 \\
\hline Postoperative death & No & No & No & No & No \\
\hline
\end{tabular}



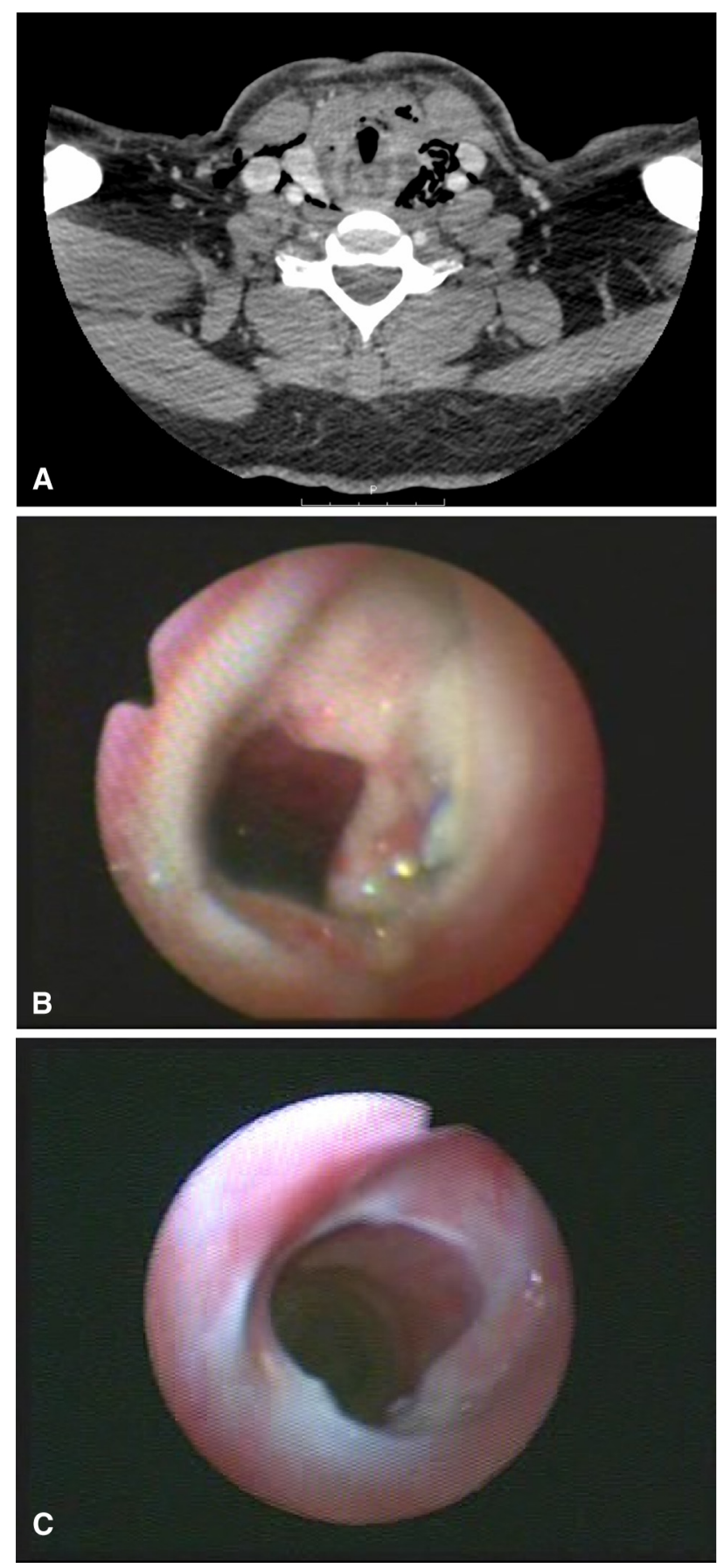

FIGURE 1. Bronchoscopy and computed tomography (CT) findings at initiation of hyperbaric oxygen therapy (HBOT) and after completion of treatment. A, Axial CT of the neck demonstrating significant subcutaneous air. B, Bronchoscopy after initiation of HBOT treatment. Note the presence of necrosis and visible suture material at the lateral aspect of the anastomosis. C, Bronchoscopy after completing 21 HBOT sessions at 48 days postoperatively. Complete healing of the anastomosis is evident. A small granuloma at the lateral aspect was noted that did not require intervention.

Bronchoscopy and axial CT are essential to evaluate the airway. Patients should be accompanied by qualified medical personnel to radiology or HBOT treatments.
Patients should also be cared for in the intensive care setting until confident that the airway is stable.

The application of HBOT increases the partial pressure of oxygen in healing tissue. ${ }^{9}$ It has been shown in randomized studies to increase the rate of healing in diabetic ulcers and prevent amputation in this patient population. ${ }^{9,10}$ Two recent studies in rats demonstrated that, after tracheal resection, animals treated with HBOT had increased rates of epithelialization, decreased inflammation, and overall increased anastomotic healing compared with control rats. 3,4

HBOT is believed to improve wound healing through several mechanisms. HBOT generates reactive oxygen and nitrogen species, which stimulate angiogenesis by local endothelial cells and through the activation of circulating stem/progenitor cells derived from the bone marrow. ${ }^{2}$ Many growth factors known to be important in angiogenesis including vascular endothelial growth factor, plateletderived growth factor, and hypoxia-inducible factor- 1 and -2 are increased after the administration of HBOT. ${ }^{2,11}$ In addition, HBOT has been shown to increase fibroblast proliferation and collagen production in healing wounds. ${ }^{12}$

Treatment protocols for HBOT are variable. HBOT is usually administered at 2 to $3 \mathrm{~atm}$ once or twice daily for 20 to 40 days. However, some studies have prolonged treatment for up to 8 weeks. ${ }^{10}$ At our institution, we favored a target of 20 treatments of HBOT delivered at 2 atm. As our experience with HBOT increased, we increased the frequency from daily treatments to twice daily treatments to reduce the duration of therapy. In our study, the average number of treatments received was 13.2. HBOT was discontinued when patients showed signs of significant anastomotic healing on bronchoscopy regardless of the number of treatments they had received.

Our results are significant in that reoperation or a permanent tracheostomy was avoided in all patients treated with HBOT. The exception to this is the patient who underwent revision of the anastomosis and tracheostomy placement before the initiation of HBOT. Significantly, this patient did not undergo buttressing of the anastomosis with strap muscle at the time of the initial operation. We routinely perform a muscle flap on most of our tracheal anastomoses and believe that the use of a muscle buttress is essential for a successful outcome with HBOT. A strap muscle buttress acts to seal the anastomosis when necrosis or separation occurs, preventing free communication between the tracheal lumen and the surrounding soft tissues. Also, the muscle flap provides additional blood supply and may work in concert with HBOT by providing a bed of relatively wellvascularized tissue on which to form granulation tissue.

Antibiotic therapy is also an important aspect in the management of these patients. Two of the 5 patients in this series had a documented infection with a pathologic organism. We favor obtaining cultures and starting broad-spectrum 
antibiotics in all patients who present with anastomotic disruptions after TRR. As a result of our experience with the lung transplant population, we began using inhaled tobramycin in patients being treated with HBOT for tracheal complications. Previously, inhaled tobramycin has been shown to improve tracheobronchial healing in patients with tracheobronchial anastomoses. ${ }^{13}$ It has been suggested that this is the result of decreased bacterial burden during the period of early ischemia after tracheobronchial anastomosis. ${ }^{13}$ There are too few patients in this series to compare the outcomes of patients who received inhaled tobramycin and those who did not.

Complications as a result of HBOT treatment are rare. The most common complication is ear pain occurring in $17 \%$ of patients in 1 series. ${ }^{14}$ Rarely, oxygen toxicity resulting in grand mal seizures occurs. ${ }^{14}$ Progressive myopia and cataract formation have also been reported. ${ }^{15,16}$ In this series, the number of complications were low and were minor. One patient developed blurry vision and required temporary bilateral tympanostomy tubes for ear pain. No deaths were observed. Three of the 5 patients developed granulation tissue at the anastomosis. However, bronchoscopic debridement was required in only 1 patient. Our initial experience with HBOT therefore demonstrates that it seems to promote tracheal healing without formation of exuberant granulation tissue.

This study describes our initial experience with HBOT for tracheal anastomotic complications. Without a larger number of patients and randomization, it is difficult to quantify the effect of HBOT, however compared with our previous experience managing anastomotic complications, we have observed a significantly decreased time course for healing to occur when patients are treated with HBOT. Previously, when anastomotic complications have been observed, we have been aggressive about securing the airway; all patients have undergone an intervention with either a T-tube, tracheostomy, or reoperation. Therefore, we cannot compare our outcomes with HBOT with the outcomes of a cohort treated with observation alone.

Future investigations are required to delineate the benefit of HBOT in the management of airway anastomotic complications. Other complications of thoracic surgery may be amenable to HBOT, such as anastomotic complications after lung transplantation, bronchoplastic procedures, or bronchopleural fistulas. The optimum number of treatments, duration of treatment, twice daily versus daily treatments will also need to be evaluated and standardized in the future.

\section{CONCLUSIONS}

HBOT may be effective in managing postoperative anastomotic complications after TRR. Inhaled nebulized tobramycin and intravenous antibiotics are believed to be important to avoid infectious complications of necrotic cartilage. Heliox should be considered if any difficulty in breathing is encountered. HBOT should be considered in managing airway complications after other bronchoplastic procedures or even in lung transplant patients with anastomotic complications. All patients must be carefully followed with CT scans and bronchoscopy to identify possible anastomotic dehiscence.

\section{References}

1. Wright CD, Grillo H, Wain J, Wong D, Donahue DM, Gaissert HA, et al. Anastomotic complications after tracheal resection: prognostic factors and management. J Thorac Cardiovasc Surg. 2004;128:731-9.

2. Thom SR. Hyperbaric oxygen - its mechanisms and efficacy. Plast Reconstr Surg. 2011;127(Suppl 1):131S-41S.

3. Celik B, Meydan AD, Kefeli M, Gülen EK, Okumus NO. The effects of hyperbaric oxygen treatment on the healing of tracheal anastomosis following irradiation in rats. Thorac Cardiovasc Surg. 2010;58:481-5.

4. Gorur R, Hahoglu A, Uzun G, Kutlu A, Turut H, Yiyit N, et al. Effects of hyperbaric oxygen therapy on wound healing after tracheal resection and end-to-enc anastomoses in rats: results of early observations. Thorac Cardiovasc Surg. 2008;56:359-62.

5. Grillo H, Donahue DM, Mathisen DJ, Wain JC, Wright CD. Postintubation tracheal stenosis: treatment and results. J Thorac Cardiovasc Surg. 1995;109: 486-93.

6. Grillo HC, Mathisen DJ, Wain JC. Laryngotracheal resection and reconstruction for subglottic stenosis. Ann Thorac Surg. 1992;53:54-63.

7. Donahue DM, Grillo HC, Wain JC, Wright CD, Mathisen DJ. Reoperative tracheal resection and reconstruction for unsuccessful repair of postintubation stenosis. J Thorac Cardiovasc Surg. 1997;114:934-9.

8. Ashiku SK, Kuzucu A, Grillo HC, Wright CD, Wain JC, Lo B, et al. Idiopathic laryngotracheal stenosis: effective definitive treatment with laryngotracheal resection. J Thorac Cardiovasc Surg. 2004;127:99-107.

9. Kessler L, Bilbault P, Ortega F, Grasso C, Passemard R, Stephan D, et al. Hyperbaric oxygen accelerates the healing rate of nonischemic chronic diabetic foot ulcers. Diabetes Care. 2003;26:2378-82.

10. Duzgun AP, Satir HZ, Ozozan O, Saylam B, Kulah B, Coskun F. Effect of hyperbaric oxygen therapy on healing of diabetic foot ulcers. J Foot Ankle Surg. 2008; 47:515-9.

11. Bonomo SR, Davidson JD, Yu Y, Xia Y, Lin X, Mustoe TA. Hyperbaric oxygen as a signal transducer: upregulation of platelet derived growth factorbeta receptor in the presence of $\mathrm{HBO} 2$ and PDGF. Undersea Hyperbar Med. 1998;25:211-6.

12. Dinar S, Agir H, Sen C, Yazir Y, Dalcik H, Unal C. Effects of hyperbaric oxygen therapy on fibrovascular ingrowth in porous polyethylene blocks implanted under burn scar tissue: an experimental study. Burns. 2008;34:467-73.

13. Ludwig C, Reidel R, Schnell J, Stoelben E. Inhalation with Tobramycin ${ }^{\circledR}$ to improve healing of tracheobronchial reconstruction. Eur J Cardiothorac Surg. 2009;35:797-800.

14. Plafki C, Peters P, Almeling M, Welslau W, Busch R. Complications and side ef fects of hyperbaric oxygen therapy. Aviat Space Environ Med. 2000;71:119-24.

15. Lyne AJ. Ocular effects of hyperbaric oxygen. Trans Ophthalmol Soc UK. 1978 98:66-8.

16. Palmquist BM, Philipson BO, Barr PO. Nuclear cataract and myopia during hyperbaric oxygen therapy. Br J Ophthalmol. 1984;68:113-7. 